\title{
In-situ Low Energy Argon Ion Source for the Improvement of EBSD Pattern Acquisition
}

\author{
$\underline{\text { Romaine Isaacs }}^{1}$, Anna Prokhodtseva $^{2}$, and Tomáš Vystavěl ${ }^{1}$ \\ 1. Thermo Fisher Scientific, Vlastimila Pecha 12, 62700 Brno, Czech Republic \\ 2. Thermo Fisher Scientific, Achtseweg Noord 5, 5651 GG Eindhoven, Netherlands
}

The localized polishing of sample surfaces using an in-situ low energy Ar ion source in the chamber of an SEM or a DualBeam system [1] has been demonstrated to be a valuable tool for TEM lamella cleaning [2]. However, its application in bulk local surface modification also has great potential where removal of hydrocarbon contamination from the sample exterior is required, or for samples that oxidize so quickly that external ion cleaning is not suitable.

Localized cleaning, using uPolisher, is achieved by navigating to the area of interest using the SEM and inserting a retractable gas delivery nozzle. The inert Ar gas flow is ionized by the electron beam inside the nozzle with bore size $\sim 30 \mu \mathrm{m}$ placed about $100 \mu \mathrm{m}$ away from the sample surface. The ions are accelerated towards the sample surface, which is biased in the range of 5 to $500 \mathrm{~V}$, where the cleaning occurs. The sample exposure to the ion beam is typically in the range of 1-3 minutes, depending on the sample and desired thickness removal. The cleaned region is generally elliptical in shape given by the geometry of the setup and consequently can be identified by a contrast change in SEM imaging. The cleaned region has a diameter $\sim 10$ to $20 \mu \mathrm{m}$, however larger areas can be easily cleaned by scanning the stage under the fixed nozzle.

ESBD signal originates from only a few tens of nanometers below the surface and therefore requires the highest quality surface preparation in order to obtain reliable patterns. Many traditional sample preparation techniques can result in poor pattern quality. Residual stresses from mechanical polishing, processing with Ga ions in the DualBeam system, contamination after chemical etching, as well as sample oxidation, can lead to unreliable results and increase the number of non-indexed points during EBSD analysis. Cleaning the surface with $\mu$ Polisher can reduce these effects, leading to a higher number of indexed points.

We have performed in-situ low energy Ar cleaning on the surface of copper. SEM imaging (Figure 1) compares the same region of interest before and after polishing. The improvement in surface quality is apparent from the removal of hydrocarbon contamination and oxide layers that has revealed the native surface. Figure 2 shows the enhanced pattern quality after the surface has been polished. Band Contrast images from the entire region, as well as Kikuchi patterns (inset) acquired in spot mode from a Fast CCD camera, before and after polishing demonstrate the improvement in pattern quality and signal to noise ratio after polishing. The sample was polished using Argon ions accelerated at $200 \mathrm{~V}$ for a duration of 2 minutes. Electron beam $20 \mathrm{kV}, 2.8 \mathrm{nA}$ was used to 
acquire EBSD patterns at $20.63 \mathrm{~Hz}$ to produce a map of resolution 363 x 356 px with a $1 \mu \mathrm{m}$ step size.

\section{References:}

[1] Mulders, J. and Trompenaars, P. 2016. An in-situ Low Energy Argon Ion Source for Local Surface Modification. European Microscopy Congress 2016: Proceedings. 453-454

[2] Prokhodtseva, A., Mulders, J., \& Vystavel, T. (2017). Applications of an in-situ Low Energy Argon Ion Source for Improvement of TEM and SEM Sample Quality. Microscopy and Microanalysis, 23(S1), 298-299. doi:10.1017/S1431927617002173

(a)

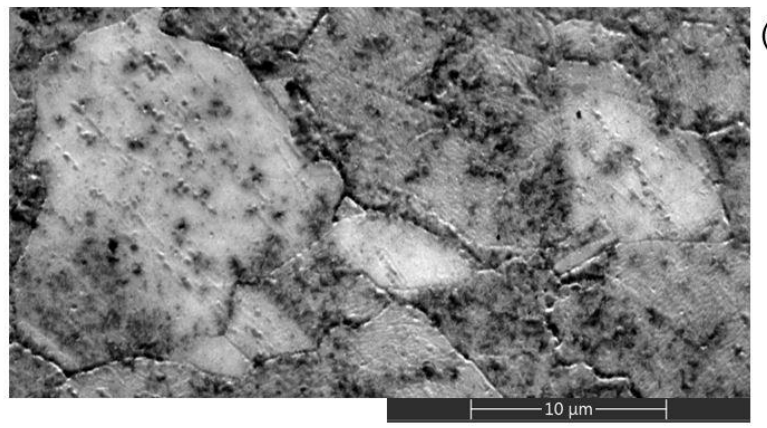

(b)

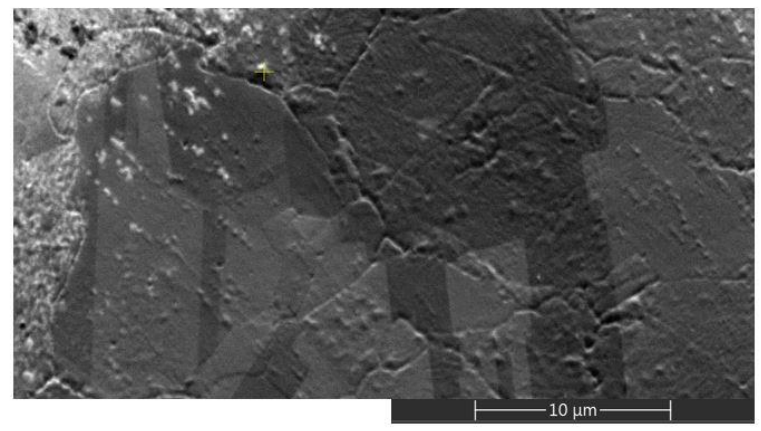

Figure 1. $\mathrm{Cu}$ surface (a) before and (b) after polishing by Ar ions using $\mu$ Polisher showing contamination removal

(a)



(b)

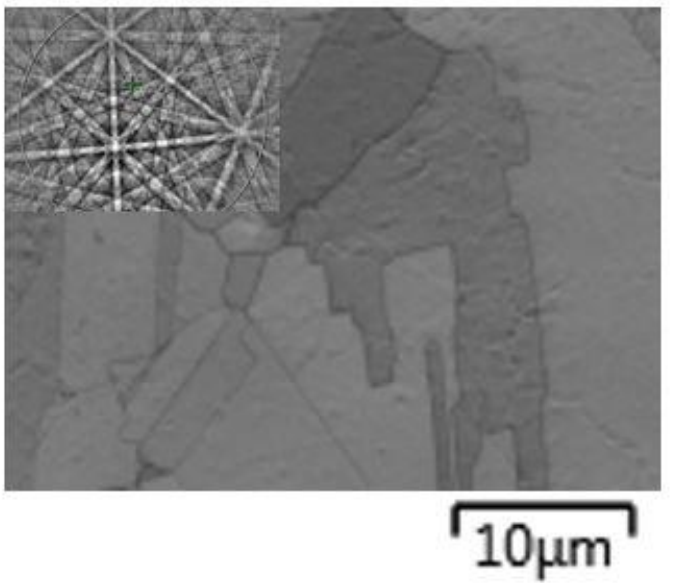

Figure 2. Band Contrast images from the same region as Figure 1 with Kikuchi pattern in insert acquired at $20 \mathrm{kV}, 2.8 \mathrm{nA}$ (a) before and (b) after polishing, showing the improvement of pattern quality 